\title{
Evaluation of intestinal injury, inflammatory response and oxidative stress following intracerebral hemorrhage in mice
}

\author{
YIJUN CHENG ${ }^{1}$, JIEYU ZAN $^{2}$, YAYING SONG ${ }^{3}$, GUOYUAN YANG $^{3,4}$, HANBING SHANG $^{1}$ and WEIGUO ZHAO ${ }^{1}$ \\ ${ }^{1}$ Department of Neurosurgery, Rui Jin Hospital, Shanghai Jiao Tong University School of Medicine, Shanghai 200025; \\ ${ }^{2}$ Department of Pediatrics, Nantong First People's Hospital, Nantong, Jiangsu 226001; ${ }^{3}$ Department of Neurology, \\ Rui Jin Hospital, Shanghai Jiao Tong University School of Medicine; ${ }^{4}$ Neuroscience and Neuroengineering \\ Research Center, Med-X Research Institute, Shanghai Jiao Tong University, Shanghai 200025, P.R. China
}

Received May 9, 2016; Accepted November 8, 2017

DOI: $10.3892 / \mathrm{ijmm} .2018 .3755$

\begin{abstract}
Intestinal injury is a common complication following intracerebral hemorrhage (ICH), which leads to malnutrition, impaired immunity and unsatisfactory prognosis. Previous studies have revealed the pathogenesis of intestinal injury following traumatic brain injury using ischemic stroke models. However, the effects of ICH on intestinal injury remain unknown. The present study aimed to investigate the pathological alterations and molecular mechanism, as well as the time course of intestinal injury following ICH in mice. Male C57BL/6 mice were randomly divided into the following seven groups ( $\mathrm{n}=6$ mice/group): Control group, which underwent a sham operation, and six ICH groups (2, 6, 12 and $24 \mathrm{~h}$, and days 3 and 7). The ICH model was induced by stereotactically injecting autologous blood in two stages into the brain. Subsequently, intestinal tissue was stained with hematoxylin and eosin for histopathological examination. Small intestinal motility was measured by charcoal meal test, and gut barrier dysfunction was evaluated by detecting the plasma levels of endotoxin. Quantitative polymerase chain reaction (qPCR), immunohistochemistry and ELISA analysis were performed to evaluate the mRNA and protein expression levels of inflammatory cytokines [interleukin (IL)-1 $\beta$, IL-6, tumor necrosis factor- $\alpha$, intercellular adhesion molecule 1 , monocyte chemotactic protein 1 and chemokine (C-C motif) ligand-5] in intestinal tissue and serum. Furthermore, intestinal leukocyte infiltration was detected by measuring myeloperoxidase activity. Oxidative stress was indirectly detected by measuring reactive oxygen species-associated markers (malondialdehyde content and superoxide dismutase
\end{abstract}

Correspondence to: Professor Hanbing Shang or Professor Weiguo Zhao, Department of Neurosurgery, Rui Jin Hospital, Shanghai Jiao Tong University School of Medicine, 197 Rui Jin Er Road, Shanghai 200025, P.R. China

E-mail: ruijinshb@qq.com

E-mail: ruijinsjwk@qq.com

Key words: intracerebral hemorrhage, intestinal injury, gut mucosa, barrier dysfunction, small intestinal motility, inflammatory response, oxidative stress activity assays) and the mRNA and protein expression levels of antioxidant genes [nuclear factor (erythroid-derived 2)-like 2, manganese superoxide dismutase and heme oxygenase 1] by qPCR and western blot analysis. The results demonstrated that significant destruction of the gut mucosa, delayed small intestinal motility, intestinal barrier dysfunction, and increased inflammatory responses and oxidative stress occurred rapidly in response to $\mathrm{ICH}$. These symptoms occurred as early as $2 \mathrm{~h}$ after $\mathrm{ICH}$ and persisted for 7 days. These findings suggested that $\mathrm{ICH}$ may induce immediate and persistent damage to gut structure and barrier function, which may be associated with upregulation of inflammation and oxidative stress markers.

\section{Introduction}

Intracerebral hemorrhage ( $\mathrm{ICH})$ is the most severe stroke subtype, and is a leading cause of morbidity and mortality (1). ICH accounts for $10-15 \%$ of all strokes in Europe, USA and Australia, and 20-30\% of all strokes in Asia; 2 million cases of ICH occur annually worldwide (2). Intestinal injury, particularly upper gastrointestinal (GI) bleeding, is a potential complication in patients with $\mathrm{ICH}$, which leads to malnutrition, impaired immunity, longer periods of hospitalization, dependence, poor outcome and even mortality (3-7). Furthermore, compared with other common complications that occur following $\mathrm{ICH}$, including pneumonia, seizures and fever, intestinal injury has received much less attention, and the associated pathophysiological alterations require further discussion and evaluation.

It is generally accepted that the intestine is the core organ of posttraumatic stress, and the initiating organ of multiple organ dysfunction in the development of severe complications under critically stressful events, including burns (8), heat stroke (9), trauma (10), brain events $(11,12)$ and transplantation (13). These stressful events may initiate a cascade of intestinal events, including destruction of the gut mucosa, barrier dysfunction, translocation of intestinal bacteria and endotoxin, and upper GI bleeding, which may lead to systemic inflammatory response syndrome (SIRS) and multiple organ dysfunction syndrome (MODS) (14). ICH is a severe type of pathological stress and a critical brain event, which may induce numerous intestinal events, as aforementioned. Following ICH, not only the general stress state, but also central nervous system damage-induced 
neural, humoral and endocrine system disorders may disrupt intestinal integrity and function. However, the underlying molecular mechanism remains to be elucidated.

To the best of our knowledge, no previous study has focused on intestinal aspects following ICH in vivo. Due to the important role of intestinal function in the prognosis of patients with $\mathrm{ICH}$, the development of effective therapeutic strategies for the treatment of intestinal complications is required. However, the pathophysiological mechanisms of ICH-induced intestinal complications are currently undefined. The present study aimed to investigate the sequentially pathological alterations and molecular mechanisms underlying ICH-induced intestinal injury.

\section{Materials and methods}

Animals and experimental groups. The present study was approved by the Laboratory Animal Ethics Committee of Rui Jin Hospital (Shanghai, China). Animal experiments were conducted in accordance with the US National Institutes of Health Guidelines (15) and followed the guidelines of the National Animal Protection of China. Male C57BL/6 mice (age, 6-8 weeks) were purchased from the Experimental Animal Center of the Chinese Academy of Sciences (Shanghai, China). Mice were maintained under specific pathogen-free conditions at a constant room temperature $\left(18-22^{\circ} \mathrm{C}\right)$ and humidity (40-70\%), under a 12-h light/dark cycle. Sterilized food and water were provided ad libitum. To evaluate the intestinal damage following $\mathrm{ICH}, 42$ mice were randomly divided into the following seven groups ( $\mathrm{n}=6 \mathrm{mice} / \mathrm{group}$ ): Control group and six ICH groups (2, 6, 12 and $24 \mathrm{~h}$, and 3 and 7 days). All efforts were made to minimize suffering and to reduce the number of mice used.

ICH mouse model. Mice were anesthetized with a single intraperitoneal dose of $100 \mathrm{mg} / \mathrm{kg}$ ketamine $/ 10 \mathrm{mg} / \mathrm{kg}$ xylazine (Sigma-Aldrich; Merck KGaA, Darmstadt, Germany). Mice were positioned in a stereotactic frame (RWD Life Science Co., Ltd., Shenzhen, China) and ICH was induced by autologous blood infusion. A $1 \mathrm{~mm}$ burr hole was drilled $2.5 \mathrm{~mm}$ lateral to the midline and $0.2 \mathrm{~mm}$ anterior to the bregma. Autologous blood $(25 \mu \mathrm{l})$ was collected from a cut in the tail using a Hamilton syringe, which was flushed with heparin prior to blood collection. A needle was advanced $3.0 \mathrm{~mm}$ into the right striatum. A total of $25 \mu \mathrm{l}$ autologous blood was injected via a double injection technique using a microinfusion pump (World Precision Instruments, Sarasota, FL, USA). An initial amount of $5 \mu \mathrm{l}$ was delivered at a rate of $1.5 \mu \mathrm{l} / \mathrm{min}$. Following a $10 \mathrm{~min}$ interval without injection, the remaining $20 \mu \mathrm{l}$ was delivered at the same rate $(1.5 \mu \mathrm{l} / \mathrm{min})$. The needle was left in place for a further $10 \mathrm{~min}$ to minimize blood backflow. Following withdrawal of the needle, the scalp was sutured. In the sham control mice, only the needle was inserted. After the operation, mice were returned back to their cages.

Small intestinal motility. Mice in each group were sacrificed at the corresponding time point. A total of $30 \mathrm{~min}$ before sacrifice, charcoal meal $(0.1 \mathrm{ml} ; 5 \%$ activated charcoal suspended in $10 \%$ aqueous gum arabic) was injected into the stomach through a gastric tube. After laparotomy, the entire small intestine was harvested. The distance traveled by the charcoal meal from the pylorus to the furthest point of small intestine was recorded. The intestinal impelling force was measured according to the following formula: Impelling force $(\%)=$ distance traveled by charcoal meal/total length of the small intestine $\mathrm{x} 100$.

Histopathology. After fixation in $10 \%$ formalin for $24 \mathrm{~h}$ at room temperature, ileal segments from each group were embedded in paraffin. Subsequently, the samples were cut into $4 \mu \mathrm{m}$ sections and were mounted onto slides. For histopathological examination, the sections were stained with hematoxylin for $10 \mathrm{~min}$ and eosin for $5 \mathrm{~min}$ at room temperature. Intestinal morphological alterations were detected by an observer blinded to the experimental group using an inverted microscope (Leica Microsystems $\mathrm{GmbH}$, Wetzlar, Germany). As described by Chiu et al (16) the histological scoring system was used to quantify the degree of intestinal damage following $\mathrm{ICH}$.

Plasma endotoxin. All blood samples were carefully obtained from the hearts of the mice prior to sacrifice and were centrifuged at $6,000 \times \mathrm{g}$ for $5 \mathrm{~min}$ at $4^{\circ} \mathrm{C}$. Endotoxin content in the plasma samples was assessed using the limulus amebocyte lysate kit (TAL, Xiamen, China) with a kinetic modification according to the manufacturer's protocol. Endotoxin concentrations were expressed as EU/ml.

Immunohistological staining. Segments of the terminal ileum were fixed in $10 \%$ neutral formalin for $24 \mathrm{~h}$ at room temperature, and $4 \mu \mathrm{m}$ sections were cut from each paraffin-embedded block. The sections were dewaxed in xylene and rehydrated, after which antigen retrieval was conducted. Briefly, the sections were immersed in citrate buffer and heated using a microwave oven for $15 \mathrm{~min}$ at $92-98^{\circ} \mathrm{C}$. Subsequently, the sections were incubated in $3 \% \mathrm{H}_{2} \mathrm{O}_{2}$ in PBS for $10 \mathrm{~min}$, and blocked in PBS containing $3 \%$ normal goat serum (Thermo Fisher Scientific, Inc., Waltham, MA, USA), $0.3 \%$ (w/v) Triton X-100 and $0.1 \%$ bovine serum albumin (Sigma-Aldrich; Merck KGaA) at room temperature for $1 \mathrm{~h}$. The sections were then incubated with a primary antibody against intercellular adhesion molecule 1 (ICAM-1; 1:100 dilution; cat. no. ab171123; Abcam, Cambridge, UK) at $4^{\circ} \mathrm{C}$ overnight. Subsequently, sections were developed with the $\mathrm{ABC}$ kit and detected with diaminobenzidine (both Vector Laboratories, Inc., Burlingame, CA, USA). The sections were then counterstained with hematoxylin, dehydrated and mounted. Histopathological alterations were observed using an inverted microscope (Leica Microsystems $\mathrm{GmbH}$ ) following a blinded procedure. Images were analyzed by Image-Pro Plus version 6.0 (Media Cybernetics, Inc., Rockville, MD, USA). Staining intensity was evaluated as follows: ' 0 ' indicates no detectable positive staining; ' 1 ' indicates very low density of positive staining; ' 2 ' indicates a moderate density of positive staining; ' 3 ' indicates higher, but not maximal, density of positive staining; '4' indicates the highest density of positive staining.

Reverse transcription-quantitative polymerase chain reaction $(R T-q P C R)$. Total RNA was extracted from intestinal samples using TRIzol reagent (Invitrogen; Thermo Fisher Scientific, Inc.) according to the manufacturer's protocol. RNA quantity was determined according to spectrophotometric analysis (optical density $\left._{260 / 280}\right)$. RT was performed using a PrimeScript RT 
Table I. Polymerase chain reaction primer sequences.

\begin{tabular}{|c|c|c|}
\hline Target gene & Sense primer (5' to $\left.3^{\prime}\right)$ & Antisense primer (5' to $\left.3^{\prime}\right)$ \\
\hline IL-1 $\beta$ & AACCTGCTGGTGTGTGACGTTC & CAGCACGAGGCTTTTTTGTTGT \\
\hline IL-6 & ACAACCACGGCCTTCCCTACTT & CACGATTTCCCAGAGAACATGTG \\
\hline $\mathrm{TNF}-\alpha$ & CAGGCGGTGCCTATGTCTC & CGATCACCCCGAAGTTCAGTAG \\
\hline ICAM-1 & CCTGATGGGCAGTCAACAGCTA & ACAGCTGGCTCCCGTTTCA \\
\hline MCP-1 & CCACAGCATGGACGAATTCA & AGCTTGCTTTGTGGCCTTCA \\
\hline CCL-5 & TCGTGCCCACGTCAAGGAGTATTT & TCTTCTCTGGGTTGGCACACACTT \\
\hline Nrf2 & CAGTCTTCACCACCCCTGAT & CTAATGGCAGCAGAGGAAGG \\
\hline MnSOD & CGTCATTCACTTCGAGCAGA & CACCTTTGCCCAAGTCATCT \\
\hline $\mathrm{HO}-1$ & ATGACACCAAGGACCAGAGC & GTGTAAGGACCCATCGGAGA \\
\hline$\beta$-actin & GTGACGTTGACATCCGTAAAGA & GCCGGACTCATCGTACTCC \\
\hline
\end{tabular}

CCL-5, chemokine (C-C motif) ligand-5; HO-1, heme oxygenase 1; ICAM-1, intercellular adhesion molecule 1; IL, interleukin; MCP-1, monocyte chemotactic protein 1; MnSOD, manganese superoxide dismutase; Nrf2, nuclear factor (erythroid-derived 2)-like 2; TNF- $\alpha$, tumor necrosis factor- $\alpha$.

reagent kit (Takara Bio, Inc., Otsu, Japan). The RT temperature protocol parameters were as follows: $37^{\circ} \mathrm{C}$ for $15 \mathrm{~min}$ and $85^{\circ} \mathrm{C}$ for $15 \mathrm{sec}$. Forward and reverse oligonucleotide primers (Table I) were designed to amplify target genes, and were synthesized by Sangon Biotech Co., Ltd. (Shanghai, China). qPCR amplification was performed on an ABI 7500 PCR instrument (Applied Biosystems; Thermo Fisher Scientific, Inc.) using SYBR Green (Takara Bio, Inc.) according to the manufacturer's protocol. PCR thermocycling conditions were as follows: 1 cycle of predenaturation at $95^{\circ} \mathrm{C}$ for $30 \mathrm{sec}$, followed by 40 cycles of denaturation at $95^{\circ} \mathrm{C}$ for $5 \mathrm{sec}$, annealing at $60^{\circ} \mathrm{C}$ for $30 \mathrm{sec}$ and extension at $72^{\circ} \mathrm{C}$ for $30 \mathrm{sec}$, and a final step at $72^{\circ} \mathrm{C}$ for $10 \mathrm{~min}$. $\beta$-actin mRNA was quantified as an endogenous control to calculate $\Delta \mathrm{Cq}$ using SDS software version 2.4.1 (Applied Biosystems; Thermo Fisher Scientific, Inc.). Results were calculated by using the $2^{-\Delta \Delta \mathrm{Cq}}$ method (17).

Western blot analysis. Total protein was extracted from intestinal samples in each group using radioimmunoprecipitation assay lysis buffer (EMD Millipore, Bedford, MA, USA) for $30 \mathrm{~min}$. Following centrifugation at $12,000 \mathrm{x} \mathrm{g}$ for $10 \mathrm{~min}$ at $4^{\circ} \mathrm{C}$, the supernatant was collected. Protein concentrations were quantified using an enhanced bicinchoninic acid (BCA) protein assay kit (Thermo Fisher Scientific, Inc.). All samples were diluted in loading buffer (Nanjing Sunshine Biotechnology Co., Ltd., Nanjing, China) and boiled at $95^{\circ} \mathrm{C}$ for $5 \mathrm{~min}$. Total protein $(20 \mu \mathrm{g})$ was separated by $10 \%$ SDS-PAGE and the fractionated proteins were electrotransferred to a polyvinylidene fluoride membrane. The membrane was blocked with 5\% skim milk for $1 \mathrm{~h}$ at room temperature and probed with the following primary antibodies: Anti-nuclear factor (erythroid-derived 2)-like 2 (Nrf2; 1:1,000 dilution; cat. no. ab137550), anti-manganese superoxide dismutase (MnSOD; 1:5,000 dilution; cat. no. ab13533), anti-heme oxygenase (HO)-1 (1:2,000 dilution; cat. no. ab189491) and anti- $\beta$-actin (1:10,000 dilution; cat. no. ab3280) (all Abcam) at $4^{\circ} \mathrm{C}$ overnight. Subsequently, the membrane was incubated with goat anti-rabbit (cat. no. HA1001-100) or goat anti-mouse (cat. no. HA1006) horseradish peroxidase-conjugated secondary antibodies (1:5,000; Hangzhou Hua'an Medical \& Health
Instruments Co., Ltd., Hangzhou, China)for $1 \mathrm{~h}$ at room temperature. Immunoreactive bands were detected using an enhanced chemiluminescence reagent (Pierce; Thermo Fisher Scientific, Inc.) and were visualized using Gel-Pro Analyzer gel image analysis software version 6.3 (Media Cybernetics, Inc.).

Myeloperoxidase (MPO) activity. The intestinal tissue homogenate was extracted from each group and the protein concentration of each supernatant was assessed as aforementioned. Tissue protein concentrations from different groups were measured using an enhanced BCA protein assay kit (Thermo Fisher Scientific, Inc.). MPO activity in tissue homogenates was analyzed using a detection kit according to the manufacturer's protocol (cat. no. A044; Nanjing Jiancheng Bioengineering Institute, Nanjing, China). MPO activity was expressed as U/mg tissue, and 1 unit of MPO activity represents the amount of enzyme degrading $1 \mu \mathrm{mol} \mathrm{H}_{2} \mathrm{O}_{2} / \mathrm{min}$ at $25^{\circ} \mathrm{C}$.

ELISA analysis. Blood samples were collected from the hearts of mice prior to sacrifice and were centrifuged at $6,000 \times \mathrm{g}$ for 5 min at $4^{\circ} \mathrm{C}$. Serum levels of interleukin (IL)- $1 \beta$ were quantified using an ELISA kit (cat. no. ab197742; Abcam) according to the manufacturer's protocol. Absorbance was measured at $450 \mathrm{~nm}$, and concentration of the target protein was determined according to the standard curve. Protein levels were expressed as $\mathrm{pg} / \mathrm{mg}$ protein.

Malondialdehyde (MDA) content and superoxide dismutase (SOD) activity. Intestinal tissue levels of oxidative stress (OS) were indirectly assessed using MDA content and SOD activity assays. Intestine samples from each group were homogenized in cold saline, with a weight-to-volume ratio of 1:9, and the homogenate was centrifuged at $12,000 \mathrm{xg}$ for $10 \mathrm{~min}$ at $4^{\circ} \mathrm{C}$. The protein concentration in the supernatant was determined using the enhanced bicinchoninic acid protein assay kit (Thermo Fisher Scientific, Inc.). Measurements of MDA content and SOD activity in tissue homogenates were determined using detection kits (cat. nos. A003-1 and A001-1-1; Nanjing Jiancheng Biological Engineering Institute) according to the manufacturer's protocol. 

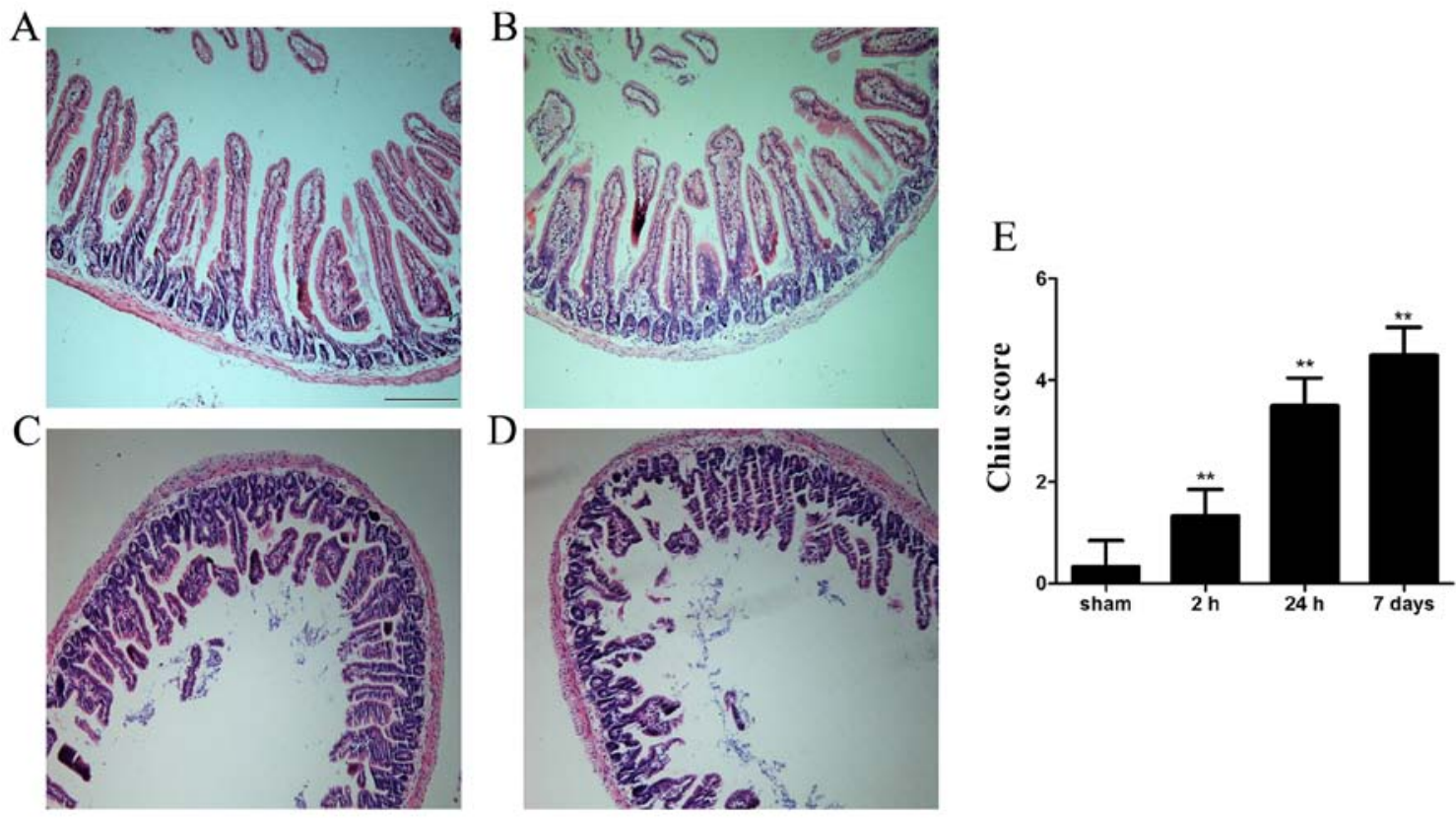

Figure 1. Hematoxylin and eosin staining of intestinal samples under light microscopy. Images were captured from the sham group and at various time-points post-ICH. Photomicrographs (magnification, x100) of the ileum of the (A) sham group, and the (B) $2 \mathrm{~h},(\mathrm{C}) 24 \mathrm{~h}$ and (D) 7 day ICH groups. (E) Chiu intestinal injury scores were determined. Scale bar, $100 \mu \mathrm{m}$. ${ }^{* *} \mathrm{P}<0.01$ vs. the sham group. ICH, intracerebral hemorrhage.

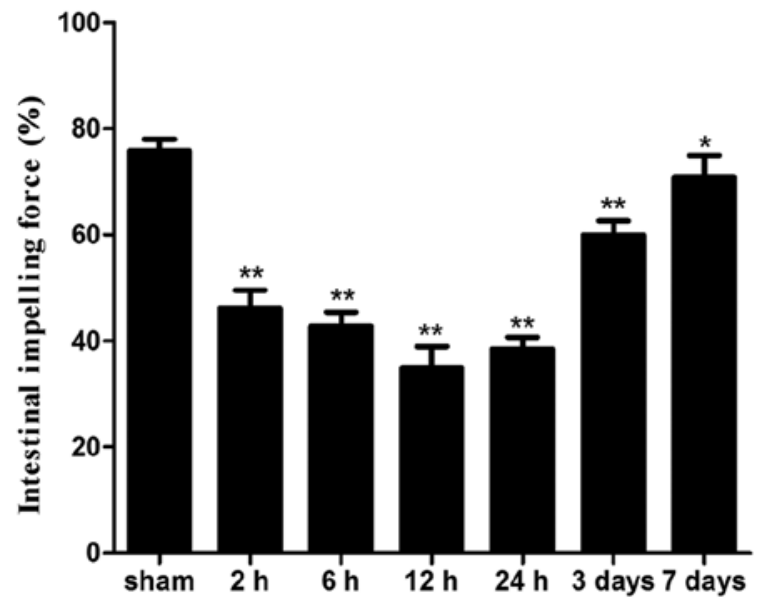

Figure 2. Effects of ICH on gastrointestinal transit in mice. Alterations in gastrointestinal transit were determined in the sham group, and in the ICH group at various time-points, by charcoal meal test. ${ }^{*} \mathrm{P}<0.05,{ }^{* *} \mathrm{P}<0.01$ vs. the sham group. ICH, intracerebral hemorrhage.

Statistical analyses. Statistical analysis was performed using SPSS 16.0 (SPSS, Inc., Chicago, IL, USA). Data were analyzed by one-way analysis of variance followed by post hoc Tukey's test. All data are representative of at least three independent experiments. Quantitative data are presented as the mean \pm standard deviation. $\mathrm{P}<0.05$ was considered to indicate a statistically significant difference.

\section{Results}

Histopathological alterations in the intestinal mucosa. Histopathological examination indicated that morphology of the intestinal mucosa was intact in the sham group. Conversely, mucosal damage was detected as early as $2 \mathrm{~h}$ after ICH; shed-

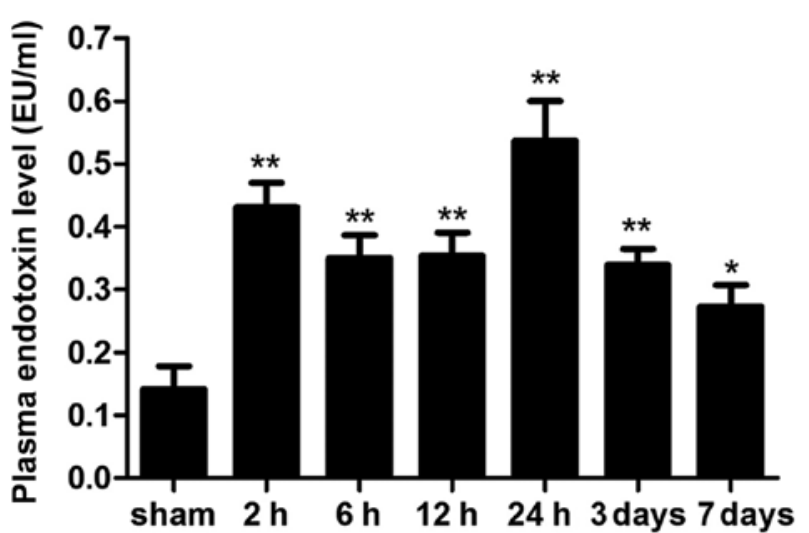

Figure 3. Determination of gut barrier dysfunction following ICH. Gut barrier function was evaluated by detecting plasma levels of endotoxin in the sham group, and in the ICH group at various time-points. Data are representative of three independent experiments. $\mathrm{P}<0.05,{ }^{* * *} \mathrm{P}<0.01$ vs. the sham group $\mathrm{ICH}$, intracerebral hemorrhage.

ding of epithelial cells from the top of villi, intestinal villi thickening and decurtation were detected. Furthermore, fusion of adjacent villi, inflammatory cell infiltration, exposed lamina propria and damaged villus tip were detected at $24 \mathrm{~h}$ and the histopathological alterations persisted for 7 days (Fig. 1).

Small intestinal motility. Intestinal impelling force was significantly lower in all ICH groups compared with in the sham group $(\mathrm{P}<0.05)$. The lowest level was detected $12 \mathrm{~h}$ after ICH and the impelling force remained significantly reduced until day 7 following ICH (Fig. 2).

Intestinal barrier dysfunction. Plasma levels of endotoxin are considered the hallmark of intestinal permeability and barrier function. Plasma endotoxin levels were markedly increased $2 \mathrm{~h}$ 

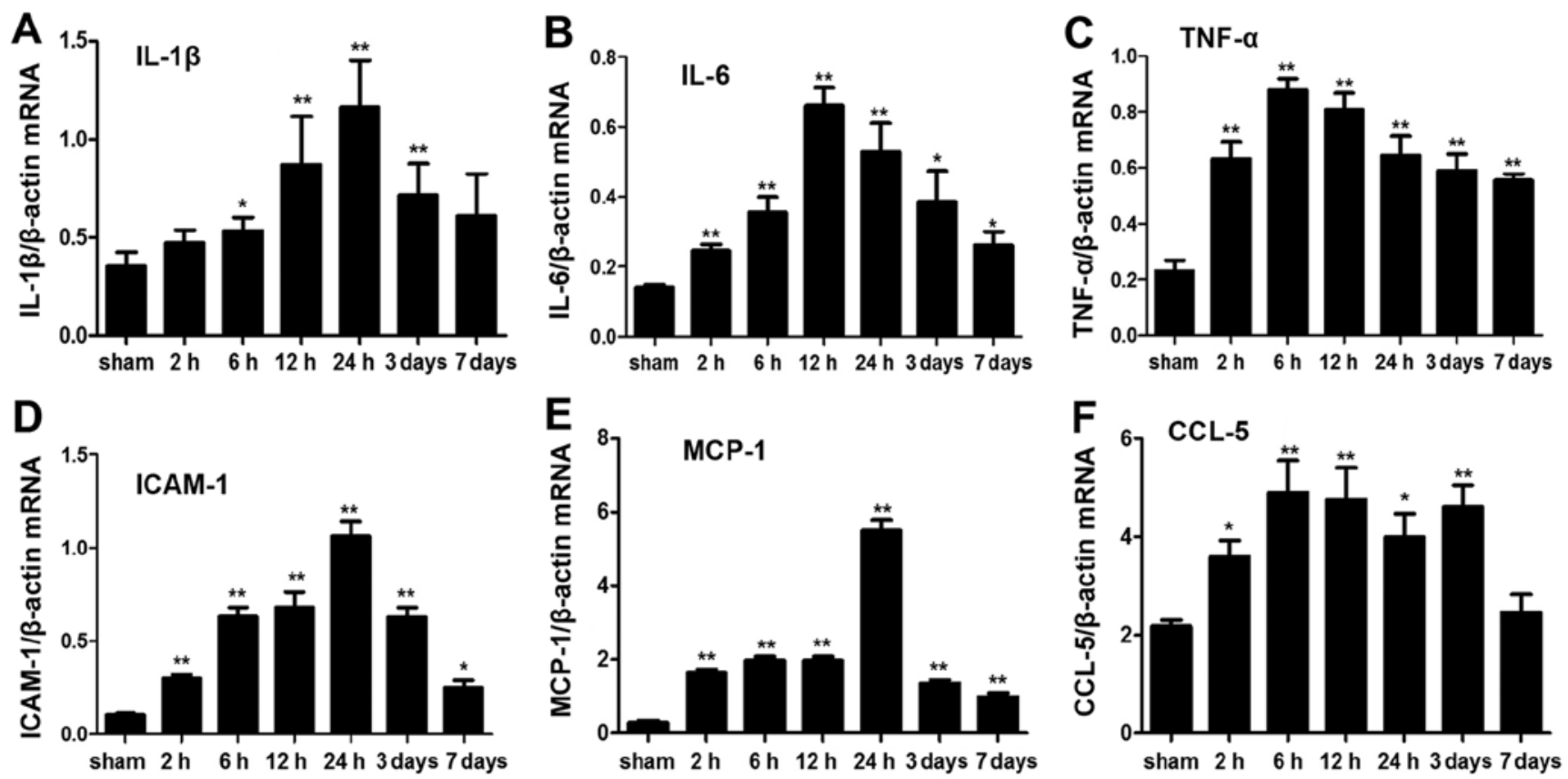

Figure 4. Analysis of inflammatory cytokine expression in intestinal tissue following ICH. (A) IL-1 $\beta$, (B) IL-6, (C) TNF- $\alpha$, (D) ICAM-1, (E) MCP-1 and (F) CCL-5 mRNA expression was detected in the sham group, and in the ICH group at various time-points, by quantitative polymerase chain reaction. $\beta$-actin served as a loading control. Data are representative of three independent experiments. ${ }^{*} \mathrm{P}<0.05,{ }^{* *} \mathrm{P}<0.01$ vs. the sham group. CCL-5, chemokine (C-C motif) ligand-5; ICAM-1, intercellular adhesion molecule 1; ICH, intracerebral hemorrhage; IL, interleukin; MCP-1, monocyte chemotactic protein 1; TNF- $\alpha$, tumor necrosis factor- $\alpha$.
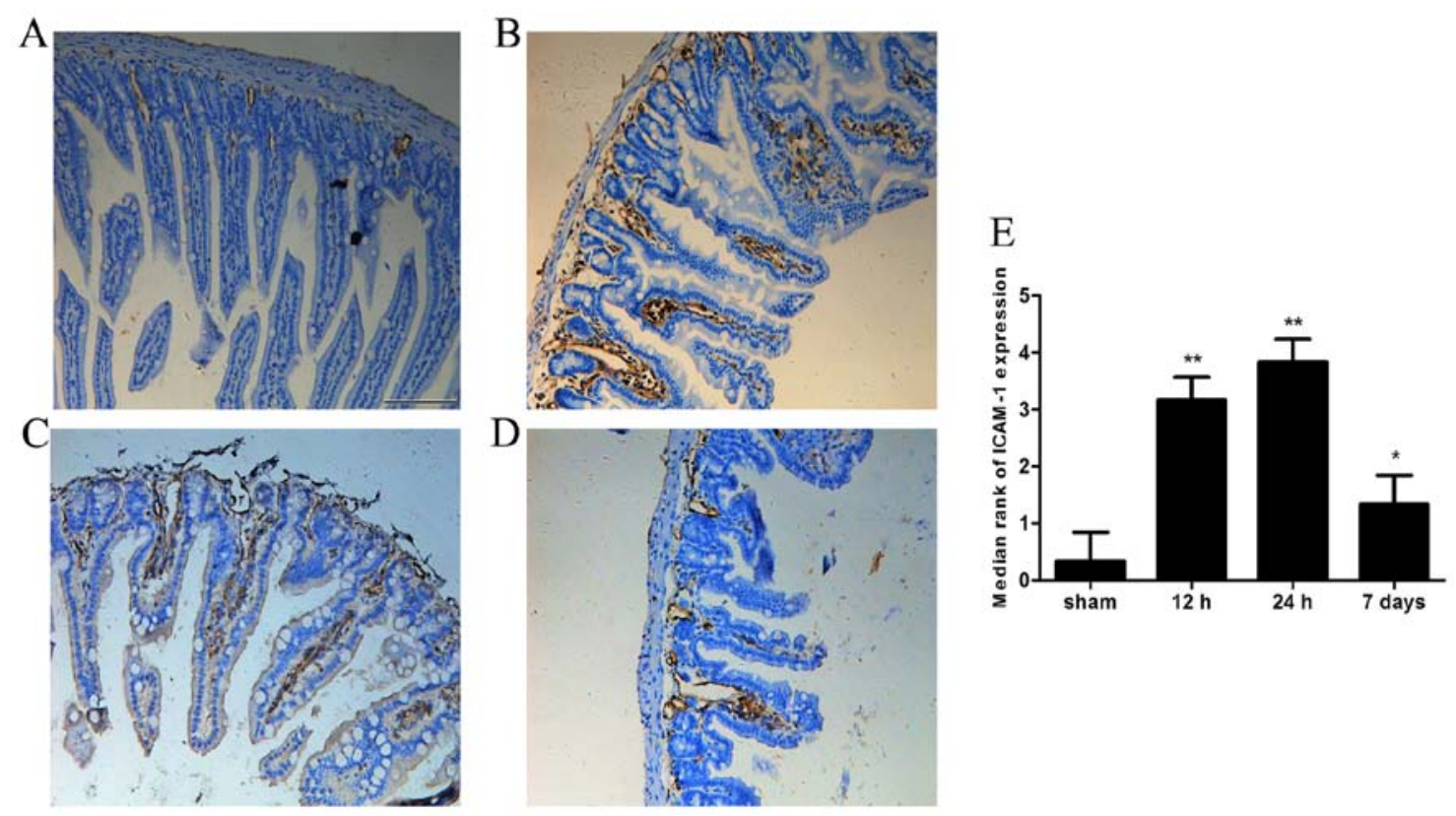

Figure 5. Detection of ICAM-1 at selected time-points by immunohistochemistry. Intestinal tissue sections from the (A) sham group, and the (B) $12 \mathrm{~h}$, (C) $24 \mathrm{~h}$ and (D) 7 day ICH groups were immunostained with anti-ICAM-1. ICAM-1 staining (brown) is visible in clusters in the intestine. (E) Staining intensity was determined. Scale bar, $100 \mu \mathrm{m}$. ${ }^{*} \mathrm{P}<0.05,{ }^{* *} \mathrm{P}<0.01$ vs. the sham group. ICAM-1, intercellular adhesion molecule $1 ;$ ICH, intracerebral hemorrhage.

following ICH, and the levels peaked at $24 \mathrm{~h}$. Notably, plasma endotoxin levels declined on day 7; however, they were still significantly higher compared with in the sham group (Fig. 3; P<0.05).

mRNA expression levels of inflammatory cytokines. The mRNA profile of intestinal inflammatory cytokines was significantly altered post-ICH. IL-1 $\beta$, IL-6 and tumor necrosis factor (TNF)- $\alpha$ are important proinflammatory cytokines, which are considered molecular markers of inflammatory responses $(18,19)$. The mRNA expression levels of IL-6 and TNF- $\alpha$ were significantly elevated after ICH at each time-point $(\mathrm{P}<0.05)$. However, the mRNA expression levels of IL- $1 \beta$ were not significantly increased $2 \mathrm{~h}$ and 7 days post-ICH $(\mathrm{P}>0.05)$. ICAM-1 is a member of the immunoglobulin superfamily, 


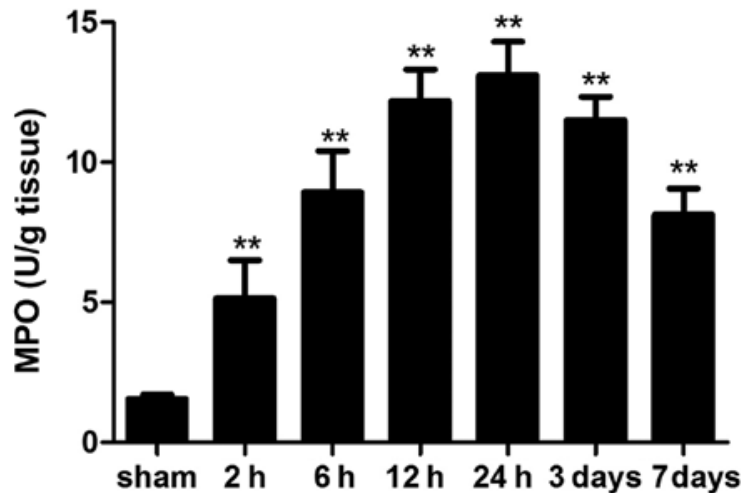

Figure 6. Effects of ICH on MPO activity in intestinal tissue. Intestinal MPO activity was detected at indicated time-points following ICH and was compared with the sham group. Data are representative of three independent experiments. ${ }^{* *} \mathrm{P}<0.01$ vs. the sham group. $\mathrm{ICH}$, intracerebral hemorrhage; MPO, myeloperoxidase.

which may initiate adhesion and infiltration of leukocytes into the lesion site, leading to damage. In the present study, ICAM-1 expression was elevated as early as $2 \mathrm{~h}$ post-ICH and was increased for 7 days $(\mathrm{P}<0.05)$. Chemokines, including monocyte chemotactic protein (MCP)-1 and chemokine (C-C motif) ligand (CCL)-5, participate in the inflammatory process by promoting the infiltration and chemotactic activation of inflammatory cells in lesions. In the present study, MCP-1 and CCL-5 in the ICH groups were also significantly elevated compared with in the sham group $(\mathrm{P}<0.05)$; however, CCL-5 mRNA expression was not significantly increased on day 7 post-ICH (Fig. 4). These results indicated that ICH may induce rapid and persistent upregulation of inflammatory cytokines in the intestine.

Expression of ICAM-1 in intestinal tissue. ICAM-1 immunoreactivity was almost undetectable in the sham group. However, strong ICAM-1 immunoreactivity was observed in the intestine 12 or $24 \mathrm{~h}$ after ICH $(\mathrm{P}<0.05)$. Furthermore, ICAM-1 immunoreactivity remained increased on day 7 post-ICH compared with in the sham group (Fig. 5).

MPO activity. MPO is an enzyme that is abundantly stored in azurophilic granules of neutrophils. MPO activity is a prognostic hallmark of inflammatory response in various acute and chronic inflammatory conditions $(20,21)$. In the present study, MPO activity was significantly increased in ICH mice compared with in the sham mice at each time-point. In addition, MPO activity on day 7 was still higher than that in the sham group (Fig. 6).

Circulating IL-1 $\beta$ levels. ICH significantly altered the circulating levels of the cytokine, IL-1 $\beta$. Following ICH, serum IL-1 $\beta$ levels were significantly elevated compared with in the sham group; however, this difference was not statistically significant at $2 \mathrm{~h}(\mathrm{P}>0.05)$. On day 7 post-ICH, serum IL-1 $\beta$ levels were still significantly elevated compared with in the sham mice (Fig. 7).

Intestinal reactive oxygen species (ROS) levels. To analyze whether ICH induces intestinal OS in mice, ROS levels were measured indirectly, by determining MDA content and SOD activity. MDA levels were significantly elevated following ICH; elevation was detected as early as $2 \mathrm{~h}$ post-ICH and remained

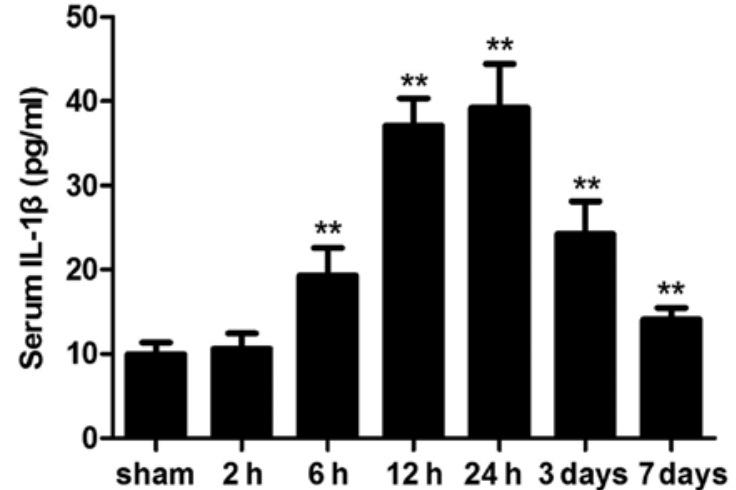

Figure 7. Alterations in circulating IL-1 $\beta$ levels at 2, 6, 12 and $24 \mathrm{~h}$, and 3 and 7 days after ICH compared with in the sham group. Serum IL-1 $\beta$ levels were significantly increased from $6 \mathrm{~h}$, peaked at $24 \mathrm{~h}$, and persisted for 7 days post- $\mathrm{ICH}$. Data are representative of three independent experiments. ${ }^{* *} \mathrm{P}<0.01$ vs. the sham group. ICH, intracerebral hemorrhage; IL-1 $\beta$, interleukin- $1 \beta$.

higher on day 7 compared with in the sham group. Conversely, SOD activity was significantly reduced by ICH (Fig. 8). These results indicated that ICH may markedly increase intestinal ROS levels in mice.

$m R N A$ and protein expression levels of antioxidants. The mRNA expression levels of the antioxidant enzymes, Nrf2, MnSOD and HO-1, were significantly increased in the intestinal samples from ICH mice compared with in the sham group, particularly at 12 and $24 \mathrm{~h}$ post-ICH. To analyze the protein levels of these antioxidant enzymes in the intestine, the protein expression levels of Nrf2, MnSOD and HO-1 were detected in the intestinal tissue of the various groups by western blot analysis. The protein expression levels of Nrf2, MnSOD and HO-1 were also markedly increased in the ICH mice compared with in the sham mice. Nrf2 and HO-1, but not MnSOD, levels remained higher in the ICH group at day 7 compared with in the sham group ( $\mathrm{P}<0.05$; Fig. 9).

\section{Discussion}

Compared with intestinal injury following ischemic stroke or traumatic brain injury (TBI), few studies have focused on intestinal injury post-ICH, particularly in experimental research. Clinically, Misra et al (4) reported that the frequency of GI bleeding post-ICH in India was 30\%, and Yang et al (5) revealed that the prevalence of GI bleeding after ICH was $26.7 \%$ in a retrospective review of 808 cases in China. Furthermore, Yang et al (5) indicated that patients with GI bleeding had significantly longer hospital stays and increased in-hospital mortality compared with patients without GI bleeding. However, the pathophysiological intestinal alterations following ICH and the molecular mechanism involved in triggering the inflammatory response and OS are unclear. On the basis of experiments in mice, the present study systematically indicated that ICH could induce significant intestinal damage, which occurred as early as $2 \mathrm{~h}$ post-ICH and lasted for 7 days. In the present study, ICH induced significant gut mucosal damage, barrier dysfunction, delayed small intestinal motility, inflammatory responses characterized by leukocyte infiltration into the intestinal tissue, elevated levels of inflammatory cytokines in intestinal tissue 

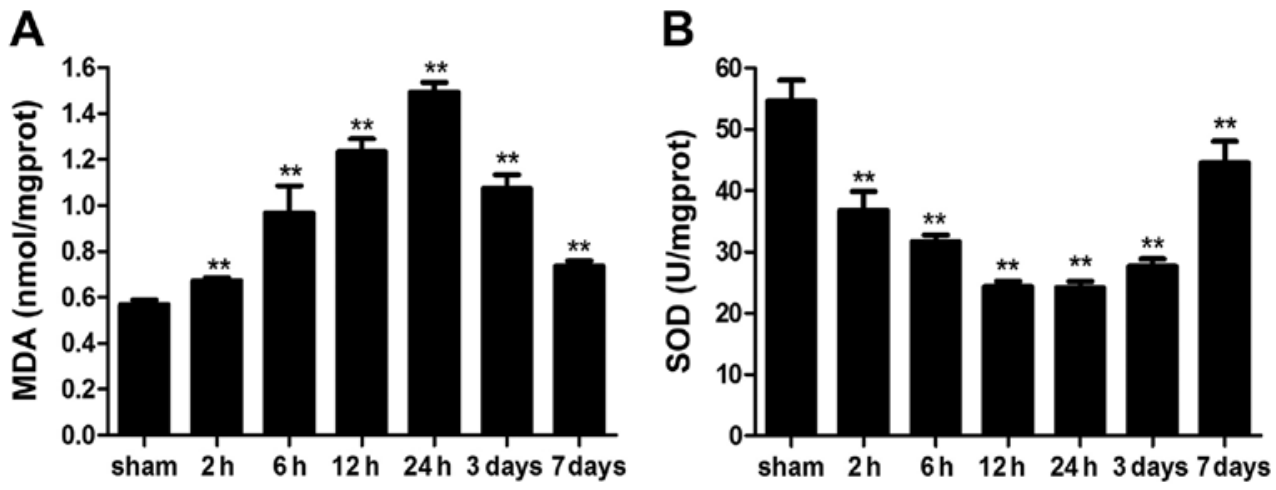

Figure 8. Measurement of oxidative stress markers following ICH. (A) MDA content and (B) SOD activity were measured in the sham group, and in the ICH group at various time-points. Data are representative of three independent experiments. ${ }^{* *} \mathrm{P}<0.01$ vs. the sham group. ICH, intracerebral hemorrhage; MDA, malondialdehyde; SOD, superoxide dismutase.

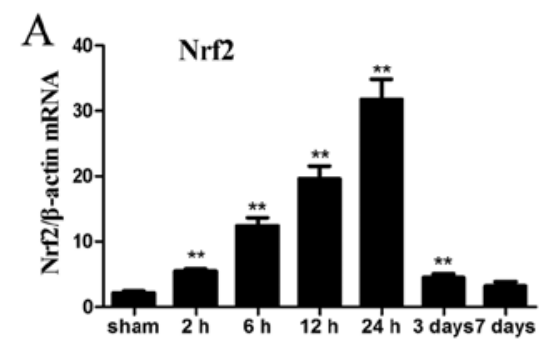

\section{B}
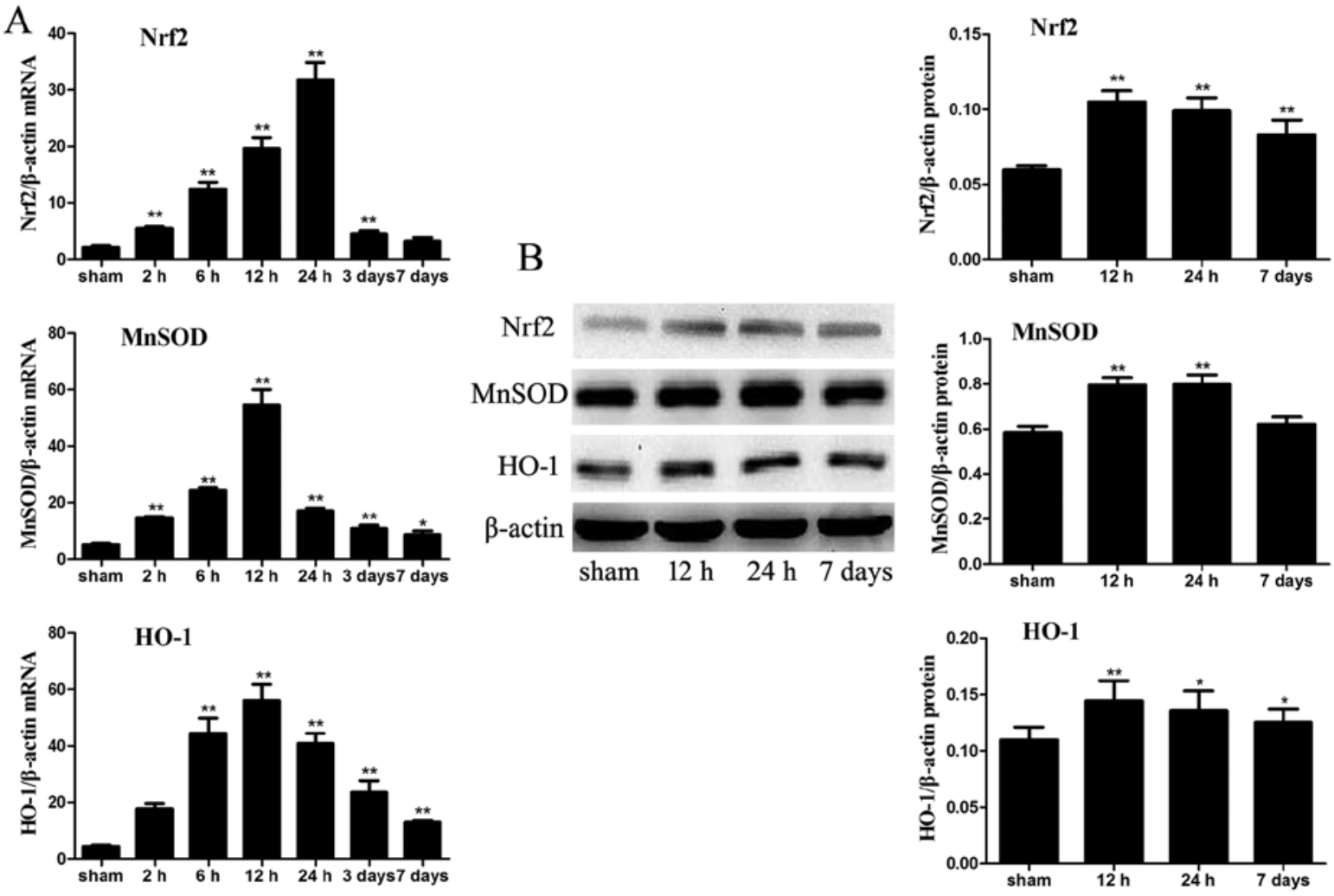

Figure 9. Analysis of antioxidant levels in intestinal tissues following ICH. (A) Quantitative polymerase chain reaction and (B) western blotting were used to detect the mRNA and protein expression levels of the following antioxidants: Nrf2, MnSOD, and HO-1. mRNA and protein expression were detected in the sham group, and in the ICH group at various time-points. $\beta$-actin served as a loading control. Data are representative of three independent experiments. ${ }^{*} \mathrm{P}<0.05,{ }^{* *} \mathrm{P}<0.01$ vs. the sham group. HO-1, heme oxygenase $1 ; \mathrm{ICH}$, intracerebral hemorrhage; MnSOD, manganese superoxide dismutase; Nrf2, nuclear factor (erythroid-derived 2)-like 2.

and serum, and OS characterized by indirect ROS levels and antioxidant gene levels.

Stressful events can induce alterations in gut mucosa, barrier function and small intestinal motility. To the best of our knowledge, no previous studies regarding intestinal mucosa structure and barrier function post-ICH have been conducted. The present study demonstrated that damage to the intestinal mucosa occurred rapidly, as early as $2 \mathrm{~h}$ post-ICH, and persisted for 7 days. The observed morphological alterations in the gut mucosa included intestinal villi engorgement, thickening, decurtation, fusion of adjacent villi, inflammatory cell infiltra- tion, exposed lamina propria and damaged villus tip. Recovery of small intestinal motility is considered a vital hallmark of clinical outcome following ICH. In a previous study, gastrointestinal motility alterations were investigated in a model of middle cerebral artery occlusion (MCAO)-induced intestinal injury; decreased small intestinal motility was confirmed within $48 \mathrm{~h}$ of MCAO (12). In the present study, the intestinal impelling ratio was significantly lower in the ICH groups compared with in the sham group. The results indicated that ICH may induce weakening in the tension of the lower esophageal sphincter, descent of gastric contraction and emptying function, and depression of 
gastrointestinal motility. In addition, gastrointestinal motility, both $2 \mathrm{~h}$ and 7 days post-ICH, was significantly lower than in the sham group, thus suggesting that intestinal dyskinesis occurs in the early stage of ICH and persists for a long period.

Impaired gut barrier function can increase intestinal permeability and translocation of endotoxin. The present study detected significantly increased plasma levels of endotoxin, with peaks detected at 2 and $12 \mathrm{~h}$ post-ICH. Hang et al (11) hypothesized the reason for this phenomenon in a TBI-induced gastrointestinal dysfunction study; it was suggested that the first peak may be due to excited sympathetic nerve-induced acute gut mucosal damage. Subsequently, the plasma levels of endotoxin may slightly decline due to recovery of liver antitoxic function and lipopolysaccharide antibodies. Finally, the second peak may be associated with severe mucosal damage, which usually occurs at later stages following a brain event. In addition, previous studies $(22,23)$ have reported that endotoxin may not only ultimately induce breakdown of the intestinal mucosal barrier, potentially resulting in lethal SIRS and MODS, but may also lead to inflammatory cell infiltration in the brain and breakdown of the blood-brain barrier via inducing production and release of proinflammatory cytokines by tissue macrophages and circulating monocytes.

To understand the molecular mechanism underlying $\mathrm{ICH}$-induced intestinal injury, the present study focused on two potential mechanisms. Firstly, the inflammatory response was analyzed by detecting the expression levels of IL-1 $\beta$, IL- 6 , TNF- $\alpha$, ICAM-1, MCP-1 and CCL-5, MPO activity in intestinal tissue, and serum levels of IL-1 $\beta$. The gut is a core organ where the inflammatory response occurs with the release of various inflammatory cytokines under stressful conditions, which has a potent impact on the small intestine. Notably, the inflammatory response may not only affect the gut itself, but may also influence the function and integrity of remote organs and tissues, including the brain, thus inducing SIRS and MODS. The gut has been labeled the 'trigger' of inflammatory responses under severe stress, which are mainly mediated by various inflammatory cytokines. Previous studies $(24,25)$ indicated that TBI could induce a rapid and persistent inflammatory response, which was associated with the upregulation of proinflammatory cytokines in the gut, including nuclear factor- $\kappa \mathrm{B}, \mathrm{TNF}-\alpha, \mathrm{IL}-6$ and ICAM-1. Consistent with these findings, a significant increase in the mRNA and protein expression levels of proinflammatory cytokines (IL-1 $\beta$, IL-6, TNF- $\alpha$, ICAM-1, MCP-1 and CCL-5) was detected using RT-qPCR, immunohistochemistry and ELISA at various time-points in the intestine and serum of ICH mice in the present study. Furthermore, detection of MPO activity confirmed that an inflammatory response had been induced. The majority of these inflammatory cytokines rapidly increased as early as $2 \mathrm{~h}$ post-ICH and persisted for 7 days. Notably, among these cytokines, IL-1 $\beta$, IL- 6 and TNF- $\alpha$ can initiate the infiltration of inflammatory cells into the intestine by activating ICAM-1 and other chemokines, including MCP-1 and CCL-5, and vice versa (26). Numerous studies have demonstrated that the release of inflammatory cytokines induces a delay in small intestinal motility, and intestinal obstruction (27-30). In addition, inflammatory cytokines exert cytotoxic effects that induce damage to microvilli, resulting in destruction of intercellular tight junctions and increased intestinal barrier dysfunction $(31,32)$. Taken together, the present study suggested that ICH could significantly increase the inflammatory response in the intestine through increased release of inflammatory cytokines, MPO activity, and peripheral circulating concentrations of IL-1 $\beta$. Therefore, the intestinal inflammatory response may be responsible for intestinal injury, and may serve an important role in the pathogenesis of gut mucosal damage, small intestinal motility and barrier dysfunction.

Secondly, the present study revealed that $\mathrm{ICH}$ triggered a pathological intestinal imbalance between oxidant and antioxidant systems. OS mediators, including ROS, which cause lipid peroxidation, protein oxidation, inhibition of mitochondrial activity, and increased membrane permeability of the cell and lysosome, lead to cell swelling/rupture. As a product of lipid peroxidation induced by ROS, MDA content can indirectly reflect the degree of OS.Under normal conditions, ROS may be cleared by antioxidants, including SOD. Redundant oxygen free radicals are generated during periods of ischemia followed by reperfusion in the $\mathrm{ICH}$-induced intestine, leading to excessive MDA accumulation and SOD consumption. In the present study, MDA levels were significantly increased in the ICH group compared with in the sham group. Conversely, SOD activity was markedly reduced. Nrf2, which is a key endogenous regulator of cellular defense against $\mathrm{OS}$, is a positive regulator of the antioxidant response element that modulates the expression of numerous genes and mobilizes the internal antioxidant system, including antioxidant genes (MnSOD and HO-1) $(33,34)$. Consistent with the aforementioned results regarding MDA and SOD, the mRNA and protein expression levels of Nrf2, MnSOD and $\mathrm{HO}-1$ were increased following $\mathrm{ICH}$ in the intestine, further indicating that OS was activated and served a critical role in ICH-induced intestinal damage. Notably, Jin et al $(35,36)$ reported that Nrf2-deficient mice were more susceptible to TBI-induced acute intestinal mucosal injury than wild-type $\mathrm{Nrf}^{+/+}$mice, as characterized by decreased intestinal mRNA expression and antioxidant enzyme activities. These findings indicated that an oxidant-antioxidant imbalance participates in the pathogenesis of ICH-induced intestinal injury.

It should be noted that OS is not a secondary event that results from the inflammatory response. OS is now thought to contribute to, rather than result from, the inflammatory response. Previous studies have reported that antioxidant genes, particularly the key upstream transcription factor Nrf2, serve a critical role in counteracting inflammation during tissue injury in various experimental models (35-38). These studies have indicated that increased Nrf2 activity has an important protective role in limiting tissue inflammatory response and injury. Notably, our previous study demonstrated that Nrf2, and its downstream antioxidant genes, were significantly increased in the $\mathrm{ICH}$ rat brain, and activation of $\mathrm{Nrf} 2$ may represent a potential target for treatment of brain injury following ICH (39). Therefore, it is hypothesized that Nrf2 may be considered a novel therapeutic target for the systematic treatment of not only ICH-induced brain damage but also intestinal injury. Further studies using Nrf2 overexpression or Nrf2 knockout mice are required to examine the exact role of Nrf2 in animal models of ICH-induced intestinal injury.

In conclusion, the results of the present study systematically demonstrated that ICH may markedly induce gut mucosal damage, barrier dysfunction, delayed small intestinal motility, inflammatory response and OS in the intestine. To the best of our knowledge, the present study is the first to elucidate the 
pathophysiological intestinal alterations that occur following ICH. Ultimately, based on a better understanding of the physiological and pathological processes of ICH-induced intestinal injury, strategies that attenuate the severe complication of intestinal injury-induced SIRS and MODS following severe ICH will be a vital therapeutic target for future ICH research.

\section{Acknowledgements}

The present study was supported by the Natural Science Fund (grant nos. 14ZR1426000 and 16ZR14212000) from the Science and Technology Commission of Shanghai Municipality.

\section{Competing interests}

The authors declare that they have no competing interests to disclose.

\section{References}

1. Keep RF,Hua Y and Xi G: Intracerebral haemorrhage: Mechanisms of injury and therapeutic targets. Lancet Neurol 11: 720-731, 2012.

2. Adeoye $\mathrm{O}$ and Broderick JP: Advances in the management of intracerebral hemorrhage. Nat Rev Neurol 6: 593-601, 2010.

3. Lu WY, Rhoney DH, Boling WB, Johnson JD and Smith TC: A review of stress ulcer prophylaxis in the neurosurgical intensive care unit. Neurosurgery 41: 416-425, discussion 425-426, 1997.

4. Misra UK, Kalita J, Pandey S and Mandal SK: Predictors of gastrointestinal bleeding in acute intracerebral haemorrhage. J Neurol Sci 208: 25-29, 2003.

5. Yang TC, Li JG, Shi HM, Yu DM, Shan K, Li LX, Dong XY and Ren TH: Gastrointestinal bleeding after intracerebral hemorrhage: A retrospective review of 808 cases. Am J Med Sci 346: 279-282, 2013.

6. Shinohara Y, Yanagihara T, Abe K, Yoshimine T, Fujinaka T, Chuma T, Ochi F, Nagayama M, Ogawa A, Suzuki N, et al III. Intracerebral hemorrhage. J Stroke Cerebrovasc Dis 20 (Suppl 4): S74-S99, 2011.

7. Wang WJ, Lu JJ, Wang YJ, Wang CX, Wang YL, Hoff K, Yang ZH, Liu LP, Wang AX and Zhao XQ; China National Stroke Registry (CNSR): Clinical characteristics, management, and functional outcomes in Chinese patients within the first year after intracerebral hemorrhage: Analysis from China National Stroke Registry. CNS Neurosci Ther 18: 773-780, 2012.

8. Li X, Hammer AM, Rendon JL and Choudhry MA: Intestine immune homeostasis after alcohol and burn injury. Shock 43 : 540-548, 2015.

9. Phillips NA, Welc SS, Wallet SM, King MA and Clanton TL: Protection of intestinal injury during heat stroke in mice by interleukin-6 pretreatment. J Physiol 593: 739-753, 2015.

10. Timmermans K, Sir Ö, Kox M, Vaneker M, de Jong C, Gerretsen J, Edwards M, Scheffer GJ and Pickkers P: Circulating iFABP Levels as a marker of intestinal damage in trauma patients. Shock 43 : 117-120, 2015.

11. Hang CH, Shi JX, Li JS, Wu W and Yin HX: Alterations of intestinal mucosa structure and barrier function following traumatic brain injury in rats. World J Gastroenterol 9: 2776-2781,2003.

12. Xu X, Zhu Y and Chuai J: Changes in serum ghrelin and small intestinal motility in rats with ischemic stroke. Anat Rec (Hoboken) 295: 307-312, 2012

13. Low G, Jaremko JL and Lomas DJ: Extravascular complications following abdominal organ transplantation. Clin Radiol 70 898-908, 2015.

14. Doig CJ, Sutherland LR, Sandham JD, Fick GH, Verhoef M and Meddings JB: Increased intestinal permeability is associated with the development of multiple organ dysfunction syndrome in critically ill ICU patients. Am J Respir Crit Care Med 158: 444-451, 1998.

15. National Research Council: Guide for the care and use of laboratory animals. 8th edition. National Academies Press, Washington DC, pp1-246, 2011.

16. Chiu CJ, McArdle AH, Brown R, Scott HJ and Gurd FN: Intestinal mucosal lesion in low-flow states. I. A morphological, hemodynamic, and metabolic reappraisal. Arch Surg 101: 478-483, 1970.
17. Livak KJ and Schmittgen TD: Analysis of relative gene expression data using real-time quantitative PCR and the 2(-Delta Delta C(T)) Method. Methods 25: 402-408, 2001.

18. Molmenti EP, Ziambaras T and Perlmutter DH: Evidence for an acute phase response in human intestinal epithelial cells. J Biol Chem 268: 14116-14124, 1993.

19. Liu XH, Yang YW, Dai HT, Cai SW, Chen RH and YeZQ: Protective role of adiponectin in a rat model of intestinal ischemia reperfusion injury. World J Gastroenterol 21: 13250-13258, 2015.

20. Arndt H, Kubes P, Grisham MB, Gonzalez E and Granger DN: Granulocyte turnover in the feline intestine. Inflammation 16: $549-559,1992$.

21. Nussbaum C, Klinke A, Adam M, Baldus S and Sperandio M: Myeloperoxidase: A leukocyte-derived protagonist of inflammation and cardiovascular disease. Antioxid Redox Signal 18: 692-713, 2013.

22. Xue B, Kasparek MS, Müller MH and Kreis ME: Modulation of intestinal afferent nerve sensitivity to inflammatory mediators following systemic endotoxin in mice. Neurogastroenterol Motil 27: 550-558, 2015.

23. Bohatschek M, Werner A and Raivich G: Systemic LPS injection leads to granulocyte influx into normal and injured brain: Effects of ICAM-1 deficiency. Exp Neurol 172: 137-152, 2001.

24. Hang CH, Shi JX, Li JS, Li WQ and Wu W: Expressions of intestinal NF-kappaB, TNF- $\alpha$, and IL-6 following traumatic brain injury in rats. J Surg Res 123: 188-193, 2005.

25. Hang CH, Shi JX, Li JS, Li WQ and Yin HX: Upregulation of intestinal nuclear factor kappa B and intercellular adhesion molecule-1 following traumatic brain injury in rats. World $\mathrm{J}$ Gastroenterol 11: 1149-1154, 2005.

26. Hang CH, Shi JX, Tian J, Li JS, Wu W and Yin HX: Effect of systemic LPS injection on cortical NF-kappaB activity and inflammatory response following traumatic brain injury in rats. Brain Res 1026: 23-32, 2004.

27. Kalff JC, Schraut WH, Billiar TR, Simmons RL and Bauer AJ: Role of inducible nitric oxide synthase in postoperative intestinal smooth muscle dysfunction in rodents. Gastroenterology 118: 316-327, 2000.

28. Wehner S, Vilz TO, Stoffels B and Kalff JC: Immune mediators of postoperative ileus. Langenbecks Arch Surg 397: 591-601, 2012.

29. The FO, de Jonge WJ, Bennink RJ, van den Wijngaard RM and Boeckxstaens GE: The ICAM-1 antisense oligonucleotide ISIS-3082 prevents the development of postoperative ileus in mice. Br J Pharmacol 146: 252-258, 2005.

30. Wehner S, Schwarz NT, Hundsdoerfer R, Hierholzer C, Tweardy DJ, Billiar TR, Bauer AJ and Kalff JC: Induction of IL-6 within the rodent intestinal muscularis after intestinal surgical stress. Surgery 137: 436-446, 2005.

31. Marchiando AM, Shen L, Graham WV, Weber CR, Schwarz BT, Austin JR II, Raleigh DR, Guan Y, Watson AJ, Montrose MH, et al: Caveolin-1-dependent occludin endocytosis is required for TNF-induced tight junction regulation in vivo. J Cell Biol 189: $111-126,2010$.

32. Turner JR: 'Putting the squeeze' on the tight junction: Understanding cytoskeletal regulation. Semin Cell Dev Biol 11: 301-308, 2000.

33. Giudice A, Arra C and Turco MC: Review of molecular mechanisms involved in the activation of the Nrf2-ARE signaling pathway by chemopreventive agents. Methods Mol Biol 647: 37-74, 2010.

34. Nguyen T, Nioi P and Pickett CB: The Nrf2-antioxidant response element signaling pathway and its activation by oxidative stress. J Biol Chem 284: 13291-13295, 2009.

35. Jin W, Wang H, Ji Y, Hu Q, Yan W, Chen G and Yin H: Increased intestinal inflammatory response and gut barrier dysfunction in Nrf2-deficient mice after traumatic brain injury. Cytokine 44: 135-140, 2008.

36. Jin W, Wang HD, Hu ZG, Yan W, Chen G, Yin HX and Yin HX: Transcription factor Nrf2 plays a pivotal role in protection against traumatic brain injury-induced acute intestinal mucosal injury in mice. J Surg Res 157: 251-260, 2009.

37. Rangasamy T, Guo J, Mitzner WA, Roman J, Singh A, Fryer AD, Yamamoto M, Kensler TW, Tuder RM, Georas SN, et al: Disruption of Nrf2 enhances susceptibility to severe airway inflammation and asthma in mice. J Exp Med 202: 47-59, 2005.

38. Lee JM and Johnson JA: An important role of Nrf2-ARE pathway in the cellular defense mechanism. J Biochem Mol Biol 37: 139-143, 2004.

39. Shang H, Yang D, Zhang W, Li T, Ren X, Wang X and Zhao W: Time course of Keap1-Nrf2 pathway expression after experimental intracerebral haemorrhage: Correlation with brain oedema and neurological deficit. Free Radic Res 47: 368-375, 2013. 